\title{
Sex of the Fetus can be Associated with Placental Pathologies in Premature Deliveries
}

Wasan Munim (MBCHB ,CABOG, FICMS) ${ }^{1}$, Niran Kamel (MBCHB,CABOG) ${ }^{2}$, Alaa Raheem Kareem(MBCHB , FICMS $)^{3}$, Mustafa Rasool Hussein Aal-Saleh $(\mathrm{MBCHB}, \text { FIBMS, FIBMS (Neph) })^{4}$, Mohammed Luay Subhi(MBCHB $)^{5}$ and Sarah Abdulkareem Ali Al-Dujaili(MBCHB $)^{6}$

\section{Abstract}

Background: There are many pregnancy complications that are significantly associated with the gender of the fetus; one of them is preterm labour, which is an important obstetric problem that may lead to many perinatal morbidity and mortality.

Objective: To find out whether there is a relation between the findings of placental pathology of premature deliveries and gender difference.

Patients and Methods: Fifty deliveries before a 32 week gestational age. Obstetrical, and placental histological findings have been compared among all males $(n=25)$, and females $(\mathrm{n}=25)$ premature neonates.

Results: The male premature fetus had distribution rate in maternal age, gestation age at the time of delivery, placental weight, and feto-placental weight-ratio in females, but a higher birthweight centile $([55.09 \pm 11.3]$ versus [43.09 \pm 8.2$])$. Histopathology of the placenta found no significant association of fetal-sex with acute inflammatory lesions $(p=0.09)$, intra-placental vascular pathological findings, or utero-placental vascular pathology. However, the chronic inflammatory lesion showed a higher pathological score in male fetuses than in the females $(\mathrm{p}=0.01)$.

Conclusion: Premature deliveries with less than thirty-two weeks, the male fetal-sex had higher placental pathological lesions, suggesting maternal immunological responses towards the invading trophoblasts. The immunological background of these pathological lesions needs further studies.

Keywords: Male fetal sex, placental pathology, premature delivery

Corresponding Author: asmaa.viro@yahoo.com

Received: $14^{\text {th }}$ May 2020

Accepted: $22^{\text {th }}$ July 2020

DOI:https://doi.org/10.26505/DJM.19025370514

\footnotetext{
${ }^{1}$ Al-Hakim General Hospital- Baghdad -Iraq

2,3,5,6 Al-Kadhimiya Teaching Hospital - Baghdad -Iraq

${ }^{4}$ Medical City Department - Baghdad Teaching Hospital -Baghdad -Iraq
} 


\section{Introduction}

Fetal sex and preterm birth: in most populations, there was more male gender among pre-term and early pre-term births including IVF births. The ratio of male-births seems to decline with the advance in gestation age. This increase in males looks to be higher in preterm births[1,2].

The highest risks of mortality in male sex during pregnancy and infancy are well identified. Males have higher fetal and neonatal mortality rates. However, the high percentage of pre-term births in males might be the explanation for higher mortality during infancy[2].

The higher male excess in pre-term births will support the explanation that depends on the processes of inducing labor that are associated with the sex of the fetus. A higher male excess occurs in medically-indicated pre-term births could explain the increased susceptibility of males to several pregnancy complications, like hypertension and growth retardation. The fetal-weight effects, or laborinducing effects of the fetal sex hormones, were most likely etiological factors [1].

The suggested mechanisms of increased risk of preterm labor in males:

1-Vascular mechanism: Placenta of male fetuses is more susceptible to severe vascular dysfunction in the form of reversed and absent umbilical artery end-diastolic blood flow. In general, it was found that male fetuses had smaller placentas than female fetuses, relative to birth weights $[2,3]$.

2-Hormonal mechanism: Serum concentration of human chorionic gonadotropin (HCG hormone) in the mothers which is regarded as a biological marker of the placental function was found to be lower in pregnancies with male fetuses. Pregnancy with a male fetus is associated with an increased risk of impaired placentation and function, which in turn underlies the higher mortality rates for males [3-5].

3- Chromosomal mechanisms: based on the difference in X-chromosome biology. In which all the $\mathrm{X}$-chromosomes are active in male fetal tissues including their placentas; either $\mathrm{x}$-chromosomes become inactive in female, or in a random fashion, this $\mathrm{x}$ chromosome could be maternal or paternal. A skewed way of the distribution of inactivation or genetic differences that escape the $\mathrm{x}$-inactivation might contribute to this hypothesis [2,5].

4- Fetal weight mechanism: Male fetuses have heavier mean body weight which might increase the possibility of PTL $[1,6]$.

5- Medical complications mechanism: Male fetuses have a higher susceptibility to some medical complications that cause by preterm births, such as infection or pregnancy induced-hypertension, which might lead to PTL [1,5].

Aim of the study to find out whether there is a difference in sex in the placental histopathology of premature labor.

\section{Patients and Methods}

A cross-sectional study was done at the department of obstetrics and Gynecology in cooperation with the department of histopathology at Al-Kadhymia Teaching hospital, from January to April 2019. Informed consent was obtained from all participating women. Fifty cases of women who expected to deliver preterm were 
enrolled in this study. Twenty-five cases were delivered male fetuses and twenty-five were delivered female fetuses. Preterm birth was anticipated on the basis of:-

1.Singleton, live-born infants, delivered between 22-32 weeks of gestation.

2.Preterm premature ruptured membrane.

3.Placental abruption and pt. with preeclampsia.

Exclusion criteria: maternal hypertension, diabetes, $\mathrm{Rh}$ sensitization, placenta previa, and hydrops.

The patients are selected and chosen according to the inclusion and exclusion criteria, and after completion of the labour, the weighing of the babies and the placentas were done and samples of the placentas are taken and put in previously prepared containers and then sent to the department of histopathology for examination. Birth weight centiles was calculated using customized birth weight percentile [6]. Pathological examination of the placental was performed by a specialized histopathologist, two samples from the umbilical cord, 2 samples of extra-placental membranes, and 4 samples of the chronic villi were prepared for examination for each case. Placental histopathological lesions were divided and scored as explained in reference [7]. The histological lesions were divided into four groups: Group I (acute inflammation), Group II (utero-placental vascular pathology), Group III (intra-placental vascular pathology) and, Group IV (lesion of chronic inflammation).

\section{Results}

A total of 50 neonates, $25(50 \%)$ were male and $25 \quad(50 \%)$ female. Regarding demographic characteristics of the study: maternal age p-value (0.6) which was not significant and, neonatal weeks of gestation $\mathrm{p}$ value (0.9) which was not significant in our research, and parity, null parity $\mathrm{p}$-value 0.4 and multiparty $\mathrm{p}$ - value 0.6 which was not significant.

The comparison between male and female gender in obstetrical history according to which preterm was anticipated were shown no significant difference in between male and female gender in preterm labour, PROM, and abruption ( $p$-value 0.6) in preeclampsia there is lower rate [2(8\%)] for male versus [3 $(12 \%)$ ] for female ( $\mathrm{p}$-value 0.7). The comparison between fetal gender regarding birth weight in grams and according to each week of gestational age which shows higher birth weight in grams of male 486 grams than female 426 grams, which was significant difference $\mathrm{p}$-value 0.04. Regarding the comparison between fetal gender birth weight centile (mean for male 55.09 \pm 11.3 ) and for female (mean $43.09 \pm 8.2$ ). And the gestational age in weeks. The birth weight centile was significantly higher for male fetuses ( $p$-value 0.04). And for the comparison between the weight of the placenta in grams between male (mean 345 grams) and the female (mean 340 grams) and the gestational age in weeks. There is no significant difference in placenta weight between male and female (p-value 0.09). The comparison between gestational age in weeks and fetoplacental weight ratio for male (mean $3.09 \pm 1.2)$ and for female $(2.88 \pm 0.9)$ which show a significant difference (p-value 0.04). Utero-placental vascular pathology $(51 \pm 0.5$ for male) $(5.4 \pm 0.6$ for female) (p-value 0.5$)$ 
and intra-placental vascular pathology (2.4 \pm 0.4 for a male) $(2.2 \pm 0.6$ for female) ( $p$ value 0.2 ) male placenta had a higher score

Table (1): The relation of histological criteria in relation to fetal sex presented as mean \pm standard deviation $\mathrm{p}$-value is predicted by two tailed test

\begin{tabular}{||l|c||c||c||}
\hline \multicolumn{1}{|c|}{ Sites of placental inflammation } & Male fetuses $(\mathrm{n}=25)$ & Female fetuses $(\mathrm{n}=25)$ & $\mathrm{P}$ value \\
\hline \hline Uteroplacental vascular pathology & $51 \pm 0.5$ & $5.4 \pm 0.6$ & 0.5 \\
\hline $\begin{array}{l}\text { Intraplacental vascular pathology } \\
\text { pathology }\end{array}$ & $2.4 \pm 0.4$ & $2.2 \pm 0.6$ & 0.2 \\
\hline
\end{tabular}

Table (2) placental chronic and acute $(2.0 \pm 0.1$ for male $)$ and $(1.2 \pm 0.09$ for inflammatory lesions in relation to fetal sex Acute inflammation ( $4.8 \pm 0.36$ for male) and $(5.3 \pm 0.4$ for female) which show no significant difference. Chronic inflammation

(gender severity) in chronic inflammation. than the placentas of females. female) (p-value 0.01). Where chronic inflammation is significantly higher in the placentas of males than females.

Table (2): Placental chronic and acute inflammation in relation to fetal sex presented as mean \pm SD pvalue is presented by two tailed test

\begin{tabular}{|l||c|c||c||}
\hline Placental inflammatory lesions & Male fetuses $(\mathrm{n}=25)$ & Female fetuses $(\mathrm{n}=25)$ & P value \\
\hline \hline Lesions of acute inflammation & $4.8 \pm 0.35$ & $5.3 \pm 0.4$ & $0.09 \mathrm{NS}$ \\
\hline Lesions of chronic inflammation & $2.0 \pm 0.1$ & $1.2 \pm 0.09$ & $0.01 \mathrm{Hs}$ \\
\hline
\end{tabular}

Table (3) shows comparison between the examination of the distribution of chronic inflammatory lesions of male and female placentas in areas interstitial trophoblast invasion of maternal deciduas (presented by the anchoring-villi and bas plate). They were more common chronic inflammation in male placenta $(2(8 \%))$ than female $(1(4 \%))$ the relative risk 1.4 and (odd ratio 2.1). There was no significant difference between the males and females, in the rate of chronic inflammation within the placental-villi.

Table (3): Lesions of chronic inflammations in comparison to fetal gender presented as number

(percent)

\begin{tabular}{|c|c|c|c|c|}
\hline Location of placental lesions & $\begin{array}{l}\text { Male fetuses } \\
\quad(n=25)\end{array}$ & $\begin{array}{l}\text { Female fetuses } \\
(\mathrm{n}=25)\end{array}$ & Relative risk & Odds ratio \\
\hline $\begin{array}{l}\text { Chronic inflammation of the basal } \\
\text { plate }\end{array}$ & $2(8 \%)$ & $1(4 \%)$ & 1.4 & 2.1 \\
\hline $\begin{array}{ll}\text { Chronic inflammation } & \text { of } \\
\text { aminiochrorion } & \end{array}$ & $2(8 \%)$ & $2(8 \%)$ & 1 & 1 \\
\hline Chronic intervillositis & $3(12 \%)$ & $4(16 \%)$ & 0.8 & 0.7 \\
\hline Chronic villitis & $1(4 \%)$ & $2(8 \%)$ & 0.7 & 0.5 \\
\hline
\end{tabular}

The comparison between male placentas in chronic uteroplacental vasculitis 2 (24\%), and female $4(16 \%)$ showed a relative risk of 1.3 and odd ratio 1.7 , which significantly differed, while there was no significant difference in decidual eosinophilia. Basal plate plasma cell differentiation shows a significant difference with the male gender 4 $(16 \%)$ than the female gender $2(8 \%)$ relation and odds ratio 2.2. 


\section{Discussion}

Frequent chronic inflammatory lesions were reported in females with recurrent miscarriage in a number of cases with chronic chorioaminionitis is reported a high rate of prematurity $[1,4]$.

Antonio Farina University of Bologona Italy[7] found spontaneous preterm labour is thought to result from the pathological and untimely activation of the common terminal pathway of the partition (uterine contractility, cervical, repining, and membrane /decidual activation, suggest that preterm parturition is a syndrome that is coursed by multiple pathogenic process, such as infection, inflammation, bleeding, and overdistention). This current study's findings suggest that in extremely preterm fetuses, there is a significantly increased chronic inflammation in male genders at the maternal-placental interphase. This result is consistent with that of Jahanfar et al [8] who reported that inflammatory cells in the chronic chorioaminionitis have distribution in the membrane similar to that seen in acute chorioaminionitis include involvement of free (exetraplacental) fetal membrane or chorionic plate and there was a tendency toward low birth weight and high frequency of preterm labour [7-10]. In this study there was an excess of basal plate plasma cells infiltration [4(16\%)], infiltrates with an increase in utero-placental vasculitis $[6(24 \%)]$, and both in male-fetal sex, however, the chronic inflammation involving intervillous-space [3(12\%)], villitis [1(4\%)] or the exetra-placental membrane [2(8\%)] were not. So, these findings suggested an increased maternal immunological-response towards the invading trophoblasts was more in the male gender in comparison with female fetuses.

This result is in agreement with that of a study who found that deciduitis might be a normal modulation of the placental allograft, or might be an abnormal immune response to it, and found that deciduitis was associated with TORCH infections that it reflects on an abnormal immunological response like SLE or it could be part of chronic inflammatory disease, and found the diagnosis of a chronicdeciduitis is similar to an assessment of the severity of the placental inflammation that depended on the extent of plasma cell infiltration[11-18]. This study group of pregnant women who delivered within less than 32 weeks confirms the excess of male fetuses and the higher birth weight centile that is associated with male sex (55.09) versus (43.09) of females as a mean as already associated with term pregnancies. However, these findings of lower rates of preeclampsia [2(8\%)] male versus [3(12\%)] female and no significant differences in the rate of abruptio-placenta [3(12\%)] for male fetuses at variance with these previously reported this could be due to low effect of fetal gender on abruptio-placenta ( $p$ - value 0.7), this indicated complex interaction between the fetal sex, complications of pregnancy and gestational age at the time of delivery [4], when the distribution of chronicplacental inflammation was examined, significantly more severe lesion was found in male than in female fetuses at the site of implantation (the area of trophoblast-invasion at the materno-decidual and materno- 
endovascular trophoblastic re-modeling) than in the placental-villi (chronic-villitis) or in the aminio-chorionic membrane (whereas invasion of interstitial trophoblast was minimal) [11-18].

\section{Conclusions}

The present study demonstrated that in premature delivery, male fetal sex is associated with more severe placental chronic inflammations at the level of the maternofetal interphase higher than the delivery of female gender, which might be suggestive of an abnormal maternal immunological response against the invading interstitial trophoblasts.

\section{Recommendations}

Gynecologists should put in mind that patients who developed premature deliveries of male fetal-sex might have higher placental pathological lesions suggesting maternal immunological responses towards the invading trophoblasts. So that full immunological workup is recommended in such cases as autoantibodies in these patients to avoid recurrent premature deliveries.

\section{References}

[1]Jennifer Zeitlin, Marie- Joseph SaurelCubizolles, Fetal sex and preterm birth: are males at greater risk? Human reproduction Vol. 17, No. 10, 2762-2768, October 2002 European society of Human reproduction and embryology.

[2]Ghidini A, Salafia CM. Gender differences of placental dysfunction in severe prematurity. BJOG. 2005;112:140-4.

[3]Andrew Edwards, Amanda Megens, Michael Peek, Evan M Wallace. Sexual orgions of placental dysfunction Lancet. 2000 January 15, 355(9199): 203-204.
[4]Spong CY, Ghidini A, Elevated secondtrimester maternal serum HCG: A marker of inadequate angiogenesis, Obstet Gynecol 1998; 91: 605-608.

[5]Ingemar Inemarsson, Gender aspects of preterm birth BJoG: An International Journal of Obstetrics and Gynecology Volume 110, Supplement 20, April 2003, Pages 34-38.

[6]Gardosi J, Francis A. Software program for the Calculation of Customized birth weight percentiles version 5.5.1,20002005published

http://www.gestation.net/Centiles/.

[7]AntonioFarinaMDabErik.S.LeShaneBSbR obertoRomero et al. High levels of fetal cellfree DNA in maternal serum: A risk factor for spontaneous preterm delivery. https://doi.org/10.1016/j.ajog.2004.12.023

[8]Jahanfar $\mathrm{S}$ and $\operatorname{Lim} \mathrm{K}$. Is there a relationship between fetal sex and placental pathological characteristics in twin gestations? Jahanfar and Lim BMC Pregnancy and Childbirth (2018) 18:285.

[9]Salafia CM, Vintzileos AM, Bantham KF, et al. Placental pathologic findings in preterm birth. Am J obstet Gynecol 1991; 165: 934938.

[10]Kolchugina GK, Morgan T. Genderbased differences in placental chorangiosis are related to increased fetal weight in males and maternal age in females. Reprod Sci G.K. Kolchugina, Pathology, Oregon Health and Science University, United States: SAGE Publications Inc.; 2011;18:212A.

[11]Salafia CM, Weigl C, Silberman L. The prevalence and distribution of acute placental inflammation in uncomplicated term pregnancies. Obstet Gynecol 1989; 73: 383 389. 
[12] Kohut K G,Anthony M N,Salafia ,C M.

Decidual and placental histologic findings in patients experiencing spontaneous abortions in relation to pregnancy order A M J.Reprod immunol 1999;37:257 -261.

[13]Al-Obaidi AB, Hussain AG, Shamran HA. Spontaneous Abortion and Failure of Human Cytotrophoblasts to adopt a vascular adhesion phenotype. J Fac Med Baghdad. 2006;48:402-406.

[14]Al-Obaidi AB, Habib MA. IFN- $\gamma$ versus IL-10 in situ expression in recurrent spontaneous abortion. IRAQI J MED SCI, 2009; VOL.7(1):21-29.

[15]Jacques SM, Qureshi F. Chronic Chorioaminionitis: a clinicopathologic and immunohistochemical study. Hum patho; 1998; 29: 1457-1461.

[16]Lorente-Pozo S, Parra-Llorca A, Torres B. Influence of Sex on Gestational Complications, Fetal-to-Neonatal Transition, and Postnatal Adaptation. Front Pediatr. 2018; 6: 6.

[17]Goldenberg RL, Andrews WW, FayePetersen OM, Goepfert AR, Cliver SP, Hauth JC. The Alabama preterm birth study: intrauterine infection and placental histologic findings in preterm births of males and females less than 32 weeks. Am J Obstet Gynecol. 2006;195:1533-7.

[18]Renzo D, Rosati A. Does fetal sex affect pregnancy Outcome ? Gender Medi. 2007;4(1):19-30. 